\title{
Improving outpatient care in pediatrics: A challenge
}

A major part of primary health care is provided through outpatient services, a long-lasting and significant tradition in medicine. To a considerable extent, these services have helped to bring the remarkable advances of the $20^{\text {th }}$ century closer to the population. Aspects such as health protection, encouragement and recommendation of healthy habits, prevention of different diseases, including infections, through specific strategies and hygiene measures, stand out, especially vaccine prescription, one of the most transcendental achievements of medicine.

Likewise, beyond basic health control, outpatient visits allow physicians to suspect or detect diseases and take the necessary measures.

However, many things have changed in medical practice in the past 50 years. There is a unanimous agreement regarding the fact that changes have not resulted in improvements for physicians or patients. The responsibilities of physicians have changed significantly, especially in relation to external factors controlling their actions, in turn influenced by the increasing commodization of health care, excessively rapid advances in medicine, an overwhelming availability of information, and technology, which has strongly invaded medical practice.

All this resulted in a progressive worsening of primary and outpatient care which may have unpredictable consequences. A recent article begins with the following phrase: "Primary care physicians are a rapidly evolving species, and in the future could become an endangered one." Such premonition does not seem exaggerated: at present, more than $20 \%$ of primary care visits in the USA are conducted by professional nurses, and this percentage is on the rise. In the first paragraphs of that article, the author asks a remarkable question on the role to be played by primary care physicians in the near future: "Will they become partners or leaders on multidisciplinary team who spending more time supervising others and less acting with patients?"1

This is an essential aspect because such deterioration has a detrimental effect on the doctor-patient relationship, and its consequences are deleterious.

To a large extent, the emergence of private health systems has had a strong influence on the relationship between doctors and patients. The pressure exerted by these systems has forced to notably reduce the time for each office visit (to approximately 10 minutes), among other things, and such conditions make it impossible to provide an adequate care. These are profitseeking companies and their actions are mainly based on having a profitable business, not on providing a good-quality health care service. Unfortunately, in Argentina, this trend has reached the medical proletariat and has greatly impacted physicians who provide outpatient care and receive meager fees for each visit. All processes across health care managed by profitseeking companies strike two victims: patients and physicians.

In terms of technology, it is worth noting that the problem is not technology itself, which will continue to advance, but the unnecessary use of such technology by physicians. This affects patients, who become anxious and feel confused, and also increases health care costs. It has been estimated that more than half of health care expenses, which are exorbitant, are allocated to ancillary studies both for hospitalized patients and outpatients, and most of these are requested on groundless assumptions.

In pediatrics, outpatient care has been traditionally one of its most important mainstays, at least since the mid- $19^{\text {th }}$ century. During office visits, the doctor-patient relationship developed in a setting where pediatricians and parents talked and listened to each other without haste. These visits helped to build children's care, not only in relation to their health, and eventually, disease, but also in relation to their development as individuals, in the present and future. This concept was wisely described by the fondly remembered Florencio Escardó. In his book La Pediatría, Medicina del Hombre, he stated: "pediatricians should always be prone to look far away, because everything they do will be reflected during the child's adulthood."

In addition, Gianantonio, our dear and alwayspresent teacher, considered that given the varying social and cultural backgrounds of children, pediatricians should have a comprehensive education, including scientific, psychological, anthropological, social and communicational aspects, that would allow them to make more effective contributions to the development of 
children and the adults they would become.

At present, outpatient care is still one of the mainstays of pediatrics, but over time, the remarkable changes I have mentioned above have impacted on our pediatric practice and, to a certain extent, have led to a decrease or loss of several of our attributes.

Leaving aside the negative external factors herein described, which I do not believe will disappear, it is appropriate and necessary that we accept the increasingly common deviations or errors observed in outpatient care. These occur in relation to different medical actions, but they are more obvious in the prescription of medications and ancillary studies, and in recommendations regarding important topics: newborn infant care, nutrition, habits, prevention, etc.

If we look backwards, pediatricians have always been very careful with medications and only prescribed a drug when it was actually necessary, and for the shortest period possible. A safe and effective drug therapy has always been considered a key strategy in pediatric health in the past century. ${ }^{2}$ Many pediatricians, especially those who have been practicing for many years, continue with this modality, which is certainly beneficial and the one we should maintain.

However, many publications indicate that, for several years, there has been a marked and unnecessary increase in medication prescription and ancillary studies use in the field of pediatrics. A recent study described drug prescription in a sample of 133800 children in France $(90 \%$ of the pediatric population). Over a one-year period, $84 \%$ of children received at least one medication, and this increased significantly in infants younger than 2 years old, with $97 \%$ of them receiving at least one medication; percentage which is absolutely alarming. ${ }^{3}$

I have dealt with the subject of antibiotic prescription in a previous editorial; ${ }^{4}$ here I will just repeat that data from different countries indicate that more than $50 \%$ of infants and toddlers with viral infections (cough, common cold, etc.) are prescribed unnecessary antibiotics. Such regrettable behavior not only provides no benefit at all, but also causes potential damage and increases bacterial resistance.

This is similar to what occurs with Attention Deficit Hyperactivity Disorder and the large number of children and adolescents who receive unnecessary medications.
Another aspect is the prescription of antipyretic agents to children with fever because it seems that temperature should be reduced "no matter what", even if it is just low-degree fever. This behavior is openly contradictory to the recognized concept that fever is a natural and beneficial response to an infection and should not be suppressed. There is no evidence that fever is harmful, even in infants and toddlers who have febrile seizures, as demonstrated in a Cochrane review. Both parents and most pediatricians are afraid of fever and this may account for their improper behavior. ${ }^{5}$ Such fear is probably based on ignorance and assuming that fever is harmful, but also on insecurity and fear of making a wrong diagnosis.

It is also worth noting that the overall quality of outpatient pediatric care has been affected. In a research study it was shown, among other things, that hardly $46 \%$ of children received adequate care and, what I believe is even more serious, a complete immunization schedule had not been prescribed in $50 \%$ of children evaluated at 2 years old. ${ }^{6}$

As we can see, in such troubled and unfavorable times for medical practice, as pediatricians we have the enormous challenge to get back on track to the most adequate outpatient care possible. If we accomplished this, many children would benefit.

José M. Ceriani Cernadas Editor in chief

http:/ /dx.doi.org/10.5546/aap.2015.eng.386

\section{REFERENCES}

1. Okie S. The evolving primary care physician. N Engl J Med 2012;366(20):1849-53.

2. Rieder M. Do we prescribe medicines rationally? Arch Dis Child 2015; Online first.

3. Bénard-Laribière A, JovéJ, Lassalle R, RobinsonP, etal. Drug use in French children: a population based reimbursement study. Arch Dis Child 2015; Online first.

4. Ceriani Cernadas JM. La indicación inadecuada e innecesaria de antibióticos en niños, un problema creciente. Arch Argent Pediatr 2015;113(1):2-3.

5. Richardson M, Purssell E. Who's afraid of fever? Arch Dis Child 2015; Online first.

6. Mangione-Smith R, DeCristofaro AH, Setodji CM, Keesey $\mathrm{J}$, et al. The quality of ambulatory care delivered to children in the United States. N Engl J Med 2007; 357(15):1515-23. 